\title{
«Studio und Archiv Paul Parin» an der Sigmund Freud PrivatUniversität Wien Paris eingerichtet
}

\author{
Johannes Reichmayr (Wien)
}

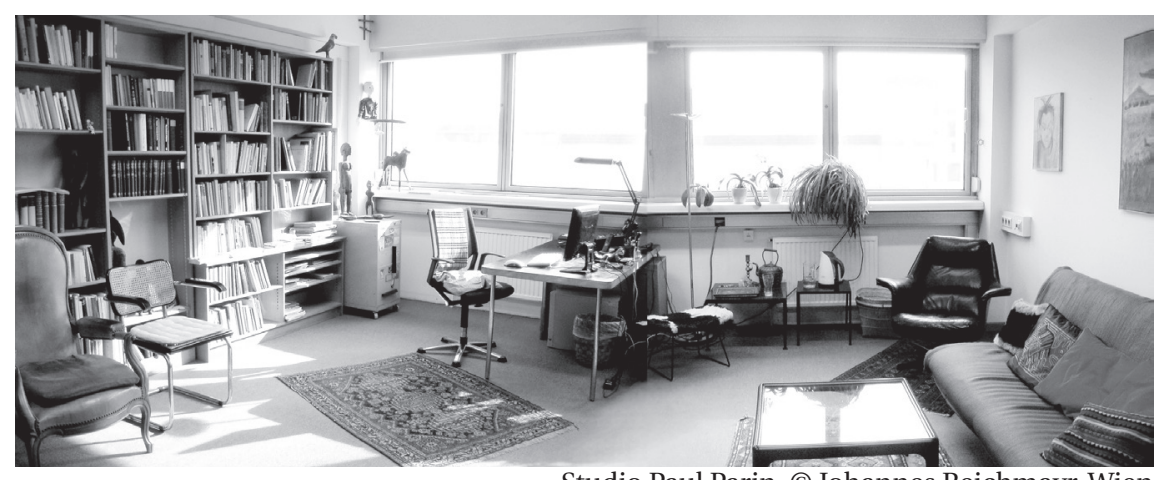

Studio Paul Parin. (C) Johannes Reichmayr, Wien

Im November 2009 übersiedelte der umfangreiche wissenschaftliche und literarische Nachlass des im Mai 2009 in Zürich verstorbenen Paul Parin an die Sigmund Freud PrivatUniversität nach Wien und wird dort bearbeitet und für die Öffentlichkeit zur Verfügung stehen. Für die Leitung der Sigmund Freud PrivatUniversität ist es eine große Freude und Ehre, den Nachlass zu beherbergen und sie hofft, mit der Bereitstellung eines Raumes und der Infrastruktur einen Beitrag dafür zu leisten, den Geist seines gesellschaftlichen, politischen, wissenschaftlichen und literarischen Engagements im Rahmen der Bearbeitung des Nachlasses lebendig zu erhalten. Paul Parin hat die Entwicklung der Sigmund Freud PrivatUniversität von Beginn an mit großem Interesse und wohlwollender Neugierde unterstützt. Das «Studio und Archiv Paul Parin» wurde dem Parin’schen Arbeitszimmer am Utoquai 41 nachgebildet und ist mit seinen Beständen eingerichtet. Für eine externe Finanzierung der archivalischen und wissenschaftlichen Bearbeitung wurde angesucht. Dieses Projekt beinhaltet neben der Inventarisierung unter anderem die Edition einer Gesamtausgabe der Schriften von Paul Parin, Goldy Parin-Matthèy und Fritz Morgenthaler, die Einrichtung einer kontinuierlich stattfindende Paul Parin-Vorlesung, die Durchführung von Ausstellungen und anderen Aktivitäten. 
Studio und Archiv Paul Parin

Sigmund Freud PrivatUniversität Wien Paris

Schnirchgasse 9A/Zimmer 509, A-1030 Wien

Tel. +43-(0) 1-798 40 98-75 / Fax: +43 (0) 1-798 40 98-20

\section{Kontakt:}

Univ.-Prof. Dr. Johannes Reichmayr

Tel.: +43-(0)6991-7872365

E-Mail: johannes.reichmayr@sfu.ac.at oder office@sfu.ac.at http:/ / www.sfu.ac.at/index.php?page $=3 \&$ article $=153$

Informationen und Gesammelte Werke von Paul Parin unter: http:/ /www.paul-parin.info 